\title{
SISTEMA AUTOMÁTICO DE MEDIÇÃO DE TEOR ALCOÓLICO EM MISTURAS ÁGUA-ÁLCOOL
}

\author{
Daniel FLORIANO \\ Danilo Alencar ALMEIDA \\ Eleandro Marcos Gomes ROSA \\ Marcelo Cristiano de SOUZA \\ Reginaldo Ferreira MENINO
}

UNIP- UNIVERSIDADE PAULISTA

Rod. Presidente Dutra, km 157,5 - Pista Sul, CEP 12240-420, São José dos Campos, SP - Brasil

Recebido em: 26/10/2015 - Aprovado em: 09/05/2016 - Disponibilizado em: 30/07/2016

\section{Resumo}

O presente artigo trata da automatização da medição de teor alcoólico em misturas água-álcool, visando sua utilização na de fabricação de aguardentes em alambiques. O dispositivo mede a variação de altura de um densímetro calibrado com uma escala de graus Gay-Lussac $\left({ }^{\circ} \mathrm{GL}\right)$, conforme sua submersão no líquido presente em uma pipeta, por meio de um sensor de distância posicionado acima do instrumento. Paralelamente, ocorre a medição de temperatura, por um sensor encapsulado presente no interior da pipeta. Um visor LCD informa o teor alcoólico da mistura. O projeto não possui precedentes no mercado brasileiro, o que o torna comercialmente interessante.

Palavras-chave:Alcoômetro, Densímetro, Misturas, Medição

\begin{abstract}
The present article deals with the automation of a measurement system for alcoholic strength by water-alcohol mixtures, aiming their use in the manufacturing process of sugar cane spirits in alembics. The product developed measures the change in height of a densimeter calibrated with a range of degrees Gay-Lussac ( ${ }^{\circ} \mathrm{GL}$ ) as the submersion in the liquid present in a pipette, by a distance sensor positioned above the instrument. Simultaneously, temperature measurement takes place by means of a sensor encapsulated inside the pipette. A LCD display report the real alcohol content of the mixture. The project is unprecedented in the brazilian market, making it commercially interesting.
\end{abstract}

Keywords: Alcoholmeter, Densimeter, Mixes, Measurement, Correction

\section{INTRODUÇÃO}

O presente trabalho teve sua origem em uma visita ao alambique Bom Jardim, situado na cidade de Jambeiro, interior do Estado de São Paulo, na Região Metropolitana do Vale do Paraíba. Todos os processos nesse alambique acontecem da forma convencional, sem qualquer automatização. $\mathrm{O}$ procedimento de destilação, fase principal de todo sistema, ocorre em um alambique de cobre, a $90^{\circ} \mathrm{C}$. O vapor produzido é condensado através do resfriamento com água corrente, e já no estado líquido, é recolhido e seu teor alcoólico analisado, após padronização. O líquido recolhido é enviado então para tonéis de envelhecimento.

Essa padronização, no entanto, gerou dúvidas quanto à sua precisão. $\mathrm{O}$ instrumento responsável pela medição do teor alcoólico do líquido resultante da destilação é um tipo de densímetro, chamado alcoômetro, que realiza medições corretas somente quando a temperatura do líquido é igual a $20^{\circ} \mathrm{C}$, sendo necessária a utilização de uma tabela de correção para qualquer temperatura diferente. Sendo assim, essa medição deve ser, incondicionalmente, acompanhada da aferição de temperatura do líquido. No estabelecimento estudado, esta medida é feita utilizando-se de um termômetro comum. 


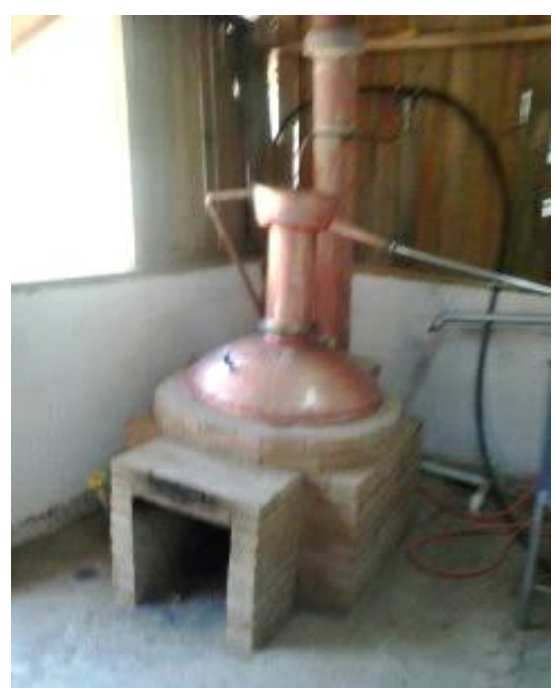

Fig. 1: Alambique de cobre

Os valores exatos de teor alcoólico medidos dependem apenas de análise a olho nu, o que pode gerar distorções nos valores. Um possível erro no valor de temperatura e teor de álcool gera erro na análise da tabela de correção, e fatalmente trará um resultado errôneo no teor alcoólico final.

A expressão matemática aproximada que padroniza o teor de álcool segundo a variação de temperatura [3] está descrita em (1):

$\mathrm{g}=\mathrm{g}_{\mathbf{0}}-\mathbf{0 , 4}\left(\mathbf{T}-\mathbf{T}_{\mathbf{0}}\right)$

As variáveis desta equação são:

- $g$ : grau alcoólico na temperatura ambiente;

- $\boldsymbol{g}_{\boldsymbol{o}}$ : grau alcoólico na temperatura de graduação do alcoômetro $\left(20^{\circ} \mathrm{C}\right)$ - valor lido no alcoômetro;

- $\boldsymbol{T}$ : temperatura ambiente;

- $\boldsymbol{T}_{\boldsymbol{o}}$ : temperatura da graduação do alcoômetro $\left(20^{\circ} \mathrm{C}\right)$;
- 0,4: constante (varia com o teor alcoólico, é mais adequada para teores alcoólicos próximos a $50 \%)$.

A solução proposta para o problema é a automatização da análise de teor alcoólico através da construção de um alcoômetro digital, utilizando como controlador central um Arduino. O sistema, contendo solução alcoólica, medirá, por meio de um sensor de distância, a tensão associada a cada posição do densímetro, que varia sua altura em função do teor alcoólico da mistura. Esta tensão, por meio de cálculos efetuados no microcontrolador, será associada diretamente ao teor alcoólico do líquido (ainda sem correção). Paralelamente, um sensor de temperatura informará suas medições ao microcontrolador. $\mathrm{O}$ sistema efetuará os cálculos de correção e informará, em um visor LCD (Liquid Crystal Display Visor de Cristal Líquido), o teor alcoólico corrigido do líquido, e sua temperatura. O modelo matemático contido em (1) estará presente na programação do dispositivo.

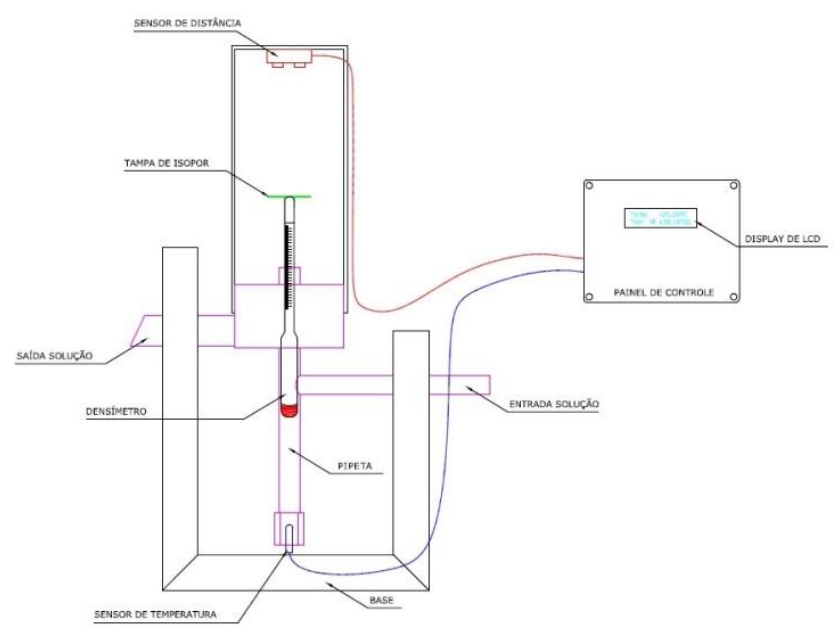

Fig. 2: Alcoômetro digital 


\section{MONTAGEM DA ESTRUTURA}

A. SENSORES E INTRUMENTOS

\section{UTILIZADOS}

- Densímetro - INCOTERM 5684;

- Sensor de distância - SHARP GP2D120;

- Sensor de temperatura - DALLAS DS18B20;

- Visor LCD - SPARKFUN ADM1602K;

- Plataforma de prototipagem - Arduino UNO.

\section{B. HARDWARE}

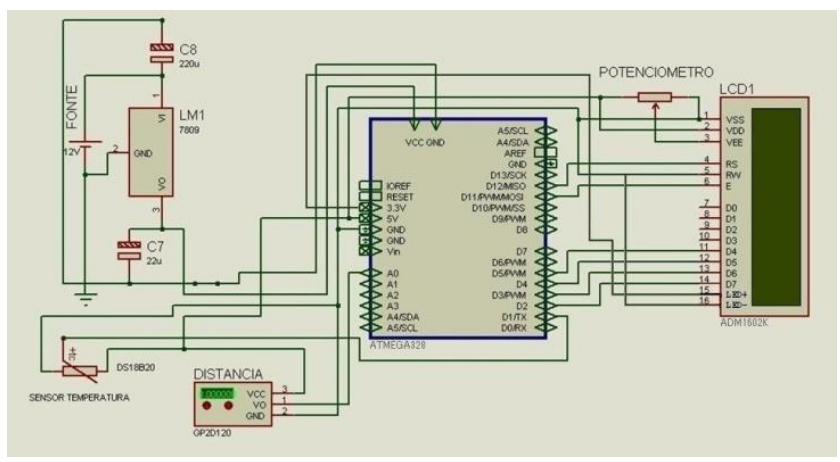

Fig. 3 - Hardware do dispositivo

A ilustração mostra o sensor de distância conectado ao microcontrolador por meio da porta A0 (analógica), o sensor de temperatura na porta D1 (digital), e o visor LCD nas portas D2 a D5 (digitais) e D11 a D12 (digitais). Foram utilizadas as portas de 3,3V e GND para a luz de fundo. Há ainda uma fonte de alimentação de $12 \mathrm{~V}$ e um potenciômetro de $10 \mathrm{k} \Omega$ ligado ao pino 3 do visor LCD, responsável por ajustar o seu contraste.
Para a fixação dos sensores, microcontrolador, visor LCD e densímetro foi construída uma estrutura fixa em alumínio que contém uma entrada e saída de líquido, uma pipeta e uma caixa contendo o visor LCD e demais dispositivos de hardware, conforme figura 4:

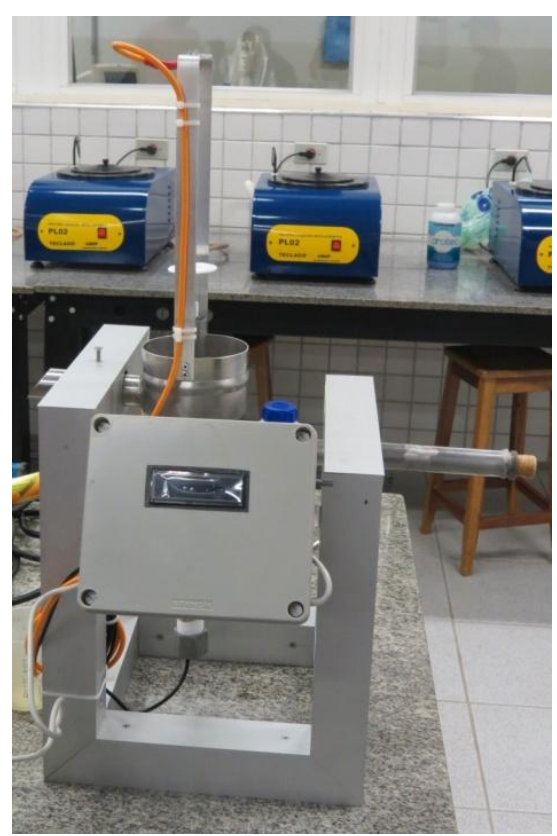

Fig. 4 - Estrutura do dispositivo

\section{PROGRAMAÇÃO}

Para a programação do microcontrolador foi utilizado o programa Arduino 1.0.4, cuja linguagem é baseada em $\mathrm{C} / \mathrm{C}++$. Ele é compatível com Windows 7, sistema operacional utilizado nos computadores nos quais a programação foi realizada. O Software (programa) é capaz de compilar no microcontrolador a programação desenvolvida e de simular resultados. 


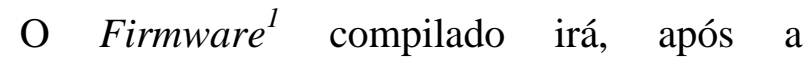
inicialização, receber os dados de tensão e com base nela calcular o teor alcoólico correspondente. Após esta etapa, será escolhido o valor de um fator $k$, chamado fator de coreção. Terminada esta escolha, serão recebidos os dados de temperatura e aplicada a equação de correção abaixo:

$g_{r}=g_{0}-k(T-20)$

Finalmente, os dados corrigidos serão enviados para o visor LCD. Após a correção do teor alcoólico, o microprograma criado retorna ao primeiro estágio, em loop (ciclo).

\section{III.RESULTADOS}

\section{A. INCERTEZA DAS MEDIÇÕES}

Utilizando-se a teoria da propagação de incertezas, o erro de medição do dispositivo pode ser dado por:

$\sigma_{g_{r}}=\sqrt{0,4761+(0,44-0,022 T)^{2}+0,25 k^{2}}$

\section{B. TESTES}

Foi realizado um teste com soluções alcoólicas entre $10^{\circ} \mathrm{GL}$ e $80^{\circ} \mathrm{GL}$, utilizando a correção de temperatura. Os dados foram lidos pelo programa Arduino 1.0.4. Foram colocadas na pipeta misturas preparadas previamente, com os teores alcoólicos desejados, medidos com um densímetro idêntico ao utilizado no projeto (INCOTERM 5684). O teste foi realizado à temperatura ambiente. Seguem, na tabela 1 , os resultados:

TABELA I - RESULTADOS DOS TESTES PARA TEMPERATURA ENTRE $17^{\circ} \mathrm{C}$ E $20^{\circ} \mathrm{C}$

\begin{tabular}{|c|c|c|c|c|c|c|c|c|c|c|}
\hline \multirow{2}{*}{$\begin{array}{c}\text { Leitura do } \\
\text { densímetro }\left({ }^{\circ} \mathrm{GL}\right)\end{array}$} & \multicolumn{4}{|c|}{ Dados experimentais } & \multirow{2}{*}{ Incerteza ( ${ }^{\circ} \mathrm{GL}$ ) } & \multicolumn{4}{|c|}{ Tabela de correção } & \multirow{2}{*}{$\begin{array}{l}\text { Leitura da } \\
\% \text { do } \\
\text { Volume } \\
\left({ }^{\circ} \mathrm{GLL}\right)\end{array}$} \\
\hline & $\left.\mathrm{g}_{0}{ }^{\circ} \mathrm{GL}\right)$ & Temperatura $\left({ }^{\circ} \mathrm{C}\right)$ & $\left.\mid \mathbf{g}_{r}{ }^{\circ} \mathbf{G L}\right) \mid$ & $\mathbf{k}\left({ }^{\circ} \mathrm{GL} /{ }^{\circ} \mathrm{C}\right)$ & & $17^{\circ} \mathrm{C}$ & $18^{\circ} \mathrm{C}$ & ${ }^{19^{\circ} \mathrm{C}}$ & ${ }_{20}^{\circ} \mathrm{C}$ & \\
\hline & 35,39 & 19,56 & 35,56 & 0.394 & 0.718 & & & 35,40 & 35,00 & 35 \\
\hline 35 & 隹 & 15,00 & & 0,374 & & & & 36,40 & 36,00 & 36 \\
\hline 40 & 41,49 & 19,31 & 41,76 & 0,394 & 0,718 & & & 40,40 & 40,00 & 40 \\
\hline & & & & & & & 45,80 & 45,40 & & $\frac{41}{45}$ \\
\hline 45 & 45,24 & 19,00 & 45,63 & 0,394 & 0,718 & & 46,80 & 46,40 & & 46 \\
\hline 50 & 50,39 & 18,94 & 50,78 & 0,367 & 0,714 & & $\begin{array}{r}50,70 \\
51,70\end{array}$ & $\frac{50,40}{51,40}$ & & $\begin{array}{c}50 \\
51\end{array}$ \\
\hline 55 & 54,13 & 18,62 & 54,64 & 0,367 & 0,715 & & $\frac{54,70}{55,70}$ & $\frac{54,40}{55,40}$ & & $\frac{54}{55}$ \\
\hline & 61,69 & 18.44 & 62,21 & 0,334 & 0.711 & & 60,70 & 60,30 & & 60 \\
\hline 60 & & & & & & & 61,70 & 61,30 & & 61 \\
\hline 65 & 65,45 & 18,00 & 66,12 & 0,334 & 0,711 & \begin{tabular}{|l|l|}
66,00 \\
67,00
\end{tabular} & \begin{tabular}{|l|l}
65,77 \\
66,70
\end{tabular} & & & $\begin{array}{l}65 \\
66\end{array}$ \\
\hline & 69,85 & 17,25 & 70,77 & 0,334 & 0,712 & 70,00 & 69,70 & & & 69 \\
\hline 70 & & & & & & $\begin{array}{l}771,00 \\
\end{array}$ & 70,60 & & & 70 \\
\hline
\end{tabular}

Pelo fato de a temperatura e o teor alcoólico $g_{0}$ não serem sempre valores inteiros, foi feita referência à tabela contida no Anexo A, contendo todos os valores de temperatura e teor alcoólico entre os quais os dados experimentais estão compreendidos. Desta forma, foi possível constatar que os teores corrigidos estavam dentro dos intervalos delimitados pela tabela utilizada como referência para o projeto.

Outro teste realizado verificou o teor alcoólico de uma aguardente industrializada, que informava $39 \%$ vol. no rótulo. A temperatura foi variada, por meio da adição inicial de gelo e elevação natural do ambiente, para que fosse analisado se haveria variação no teor alcoólico corrigido. Seguem os resultados na tabela 2:

${ }^{1}$ Também chamado microprograma, é um pequeno programa implementado em hardware com algumas característica de software [6]. 
TABELA II - RESULTADO DOS TESTES COM

AGUARDENTE 39\% VOL.

\begin{tabular}{|l|l|l|l|l|}
\hline $\mathrm{g}_{\mathbf{0}}\left({ }^{\circ} \mathrm{GL}\right)$ & $\mathrm{k}\left({ }^{\circ} \mathrm{GL} /{ }^{\circ} \mathrm{C}\right)$ & Temperatura $\left({ }^{\circ} \mathrm{C}\right)$ & $\mathrm{g}_{\mathrm{r}}\left({ }^{\circ} \mathrm{GL}\right)$ & Incerteza $\left({ }^{\circ} \mathrm{GL}\right)$ \\
\hline 36,33 & & 0,394 & &
\end{tabular}

\begin{tabular}{|l|c|c|c|c|}
\hline 36,33 & 0,394 & 15,88 & 37,95 & 0,72 \\
\hline 36,33 & 0,394 & 15,94 & 37,93 & 0,72 \\
\hline 36,33 & 0,394 & 16,06 & 37,88 & 0,72 \\
\hline 38,20 & 0,394 & 20,06 & 38,18 & 0,72 \\
\hline 38,67 & 0,394 & 20,12 & 38,62 & 0,72 \\
\hline 38,67 & 0,394 & 20,19 & 38,60 & 0,72 \\
\hline \multicolumn{4}{|c|}{ Média $g_{r}:$} \\
& Desvio padrão $g_{r}:$ & $38,19^{\circ} \mathrm{GL}$ \\
\multicolumn{5}{|c|}{$0,339^{\circ} \mathrm{GL}$} \\
\hline
\end{tabular}

\section{IV.CONCLUSÃO}

O primeiro teste realizado mostrou que os valores experimentais de $g_{r}$ ficaram contidos no intervalo determinado pela tabela de correção, sendo este um indicativo de que o projeto adotou um equacionamento aceitável. Já o experimento com cachaça industrializada apresentou uma proximidade razoável do teor alcoólico medido em relação ao informado no rótulo, tendo sido a incerteza da média de todas as medições menor que a incerteza de cada uma delas. Os resultados dos testes corroboram a idéia de que é possível e viável medir automaticamente o teor alcoólico de uma mistura sem depender de mecanismos externos.

Conhecendo-se os parâmetros necessários, qualquer densímetro pode ser utilizado na estrutura criada, utilizando os modelos matemáticos aqui presentes, e realizando as correspondentes alterações na programação.

\section{REFERÊNCIAS}

[1]ARDUINO. Arduino Uno. Disponível em:

<http://arduino.cc/en/Main/arduinoBoardUno〉. Acesso em: 4 mar. 2013

[2] BARBETTA, Pedro A; REIS, Marcelo M.; BORNIA, Antonio C. Estatística para Cursos de Engenharia e Informática. São Paulo: Editora Atlas S.A., 2010.

[3] BRAGA, Eliane C. Arantes. FísicoQuímica: Apostila de aulas práticas. Disponível em:

<http://www.fcfrp.usp.br/dfq/FQ/Apostila\%20p raticas\%202009.doc>. Acesso em 05 mar. 2013.

[4] DALLY, James W.; RILEY, Willian F.; McCONNEL, G. Kenneth. Instrumentation for Engineering Measurements. New York: John Wiley \& Sons, Inc, 1993.

[5] Instruções de uso do Alcoômetro segundo Gay Lussac. Disponível em:

<http://www.incoterm.com.br/download_anexo/ 5684_MANUAL.pdf $>$. Acesso em: 11 mar 2013.

[6] MURDOCA, Miles J.; HEURING, Vincent P. Introdução à Arquitetura de Computadores. Rio de Janeiro: Editora Campus, 2000

[7] TOGINHO FILHO, D. O.; ANDRELLO, A.C. Medição e Propagação de erros - Catálogo de Experimentos do Laboratório Integrado de Física Geral. Departamento de Física da Universidade Estadual de Londrina, 2009.

[8] UNIVERSIDADE FEDERAL DE SANTA CATARINA. Departamento de Física.

Densímetro. Disponível em:

<http://www.fsc.ufsc.br/ canzian/bau/densidad e/densidade-densimetro.html>. Acesso em: 20 fev. 2013. 